\title{
Un grand méconnu: Gustave Roussy
}

Par René Bernoulli

Le $1^{\text {er }}$ octobre 1921 est une des dates historiques de la lutte contre le cancer. Ce fut ce jour-là, à l'Hospice Paul-Brousse de Villejuif, localité de la banlieue Sud de Paris, que le directeur donna pour la première fois une consultation uniquement destinée à des cancéreux. Ce médecin, qui par ses travaux scientifiques jouissait d'une solide réputation dans le monde médical, s'appelait Gustave Roussy ${ }^{1}$. «Le visage basané, une barbe drue, coupée court, la chevelure divisée par une raie impeccable, des yeux gris d'acier, dont le regard s'illuminait facilement au contact ou au cours d'une discussion, un corps élancé et flexible sous un vêtement de bonne coupe, mais sans vaine recherche, tel était Gustave Roussy $"{ }^{2}$ selon le portrait esquissé par le professeur Denoix, son ancien assistant et deuxième successeur à la direction de l'Institut du Cancer.

Gustave Roussy était le descendant d'une famille dont les origines se situent sur les rudes plateaux des Cévennes. Lors de la révocation de l'édit de Nantes par Louis XIV en 1685, ses ancêtres se réfugièrent au pays de Vaud. C'est ici, à Vevey, que Gustave naquit le 24 novembre 1874. Par sa naissance, Roussy est donc notre compatriote. En raison de son orientation ultérieure vers la France son souvenir s'est, pourtant, quelque peu effacé en Suisse. Les mérites scientifi, ques que ce grand médecin s'est acquis sa vie durant, justifient cependant qu'onle tire de l'oubli dans lequel il fut finalement jeté par un destin tragique.

En effet, après avoir passé ses premiers semestres à la Faculté de médecine de Genève $^{3}$, Roussy s'installa, encore jeune, à Paris où il poursuivit l'étude de la médecine. Parmi ses professeurs parisiens, il signale particulièrement FrançoisFranck, Darier, Dejerine et Marie ${ }^{4}$, membres de la Faculté qui, sans conteste, ont contribué à l'évolution de la médecine. Selon ses propres aveux, c'étaient ces médecins qui lui apprirent «à connaître les ressources de la médecine expérimentale et la nécessité de la méthode dans l'interprétation des faits d'observation ${ }^{5}$, principes de recherche que la médecine doit surtout à Claude Bernard. C'est donc à ces maîtres que Roussy doit son orientation scientifique qui «a été sans cesse guidée par deux idées directrices: l'une physio-pathologique, l'autre anatomoclinique ». ${ }^{6}$ Voilà les éléments qui ne cessèrent d'influencer profondément sa carrière de médecin et de savant. Ajoutons que le praticien préconisait l'unité personnelle et locale de la recherche et de la clinique. 


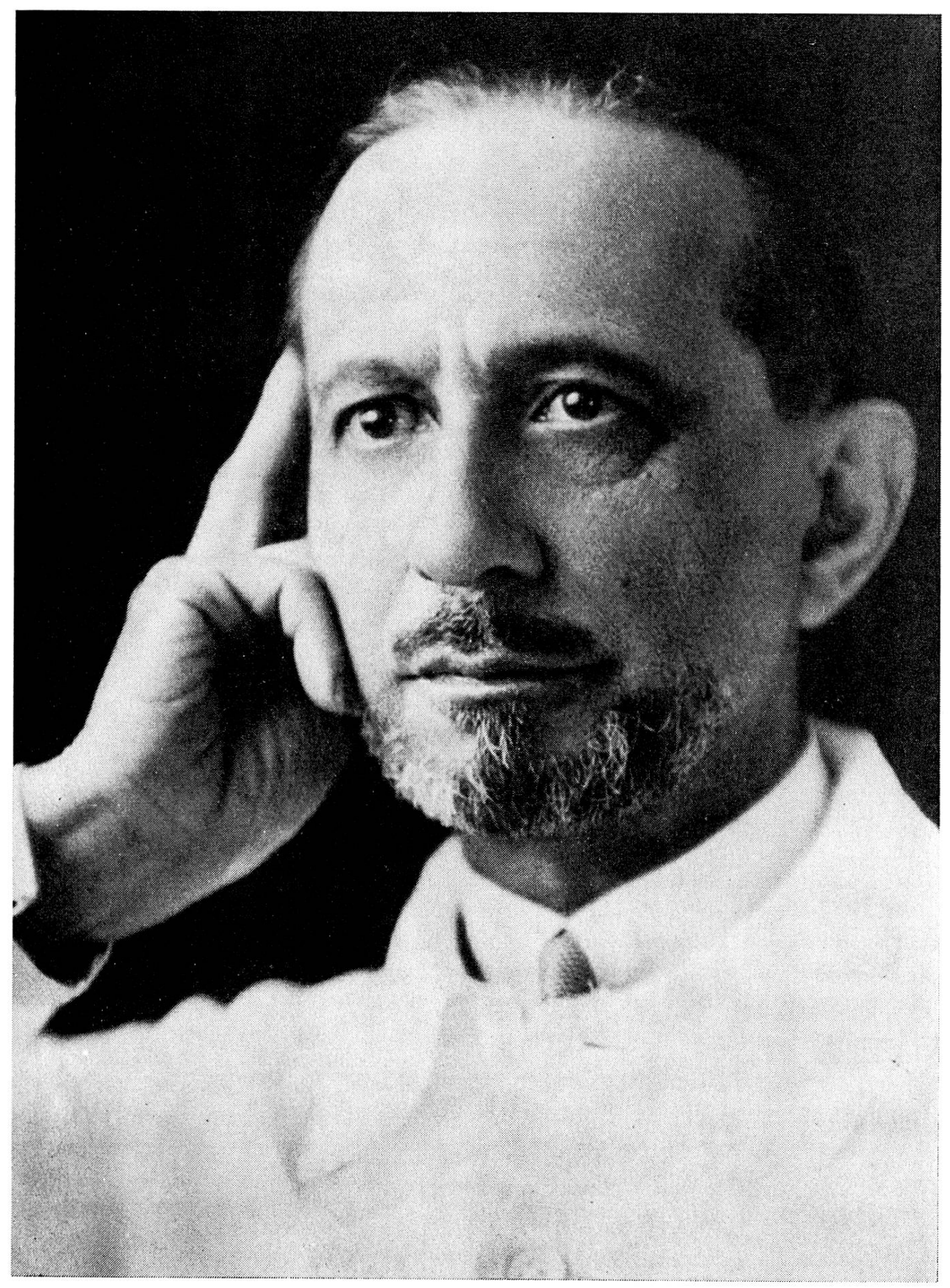

Gustave Roussy

(Photographie gracieusement communiquée par le Professeur Denoix) 
Grâce à ses talents, le jeune médecin suisse se fit apparemment vite remarquer par ses patrons ? En 1899, âgé de 25 ans, le voici déjà externe des Hôpitaux de Paris; il fut promu interne deux ans plus tard et occupa ce poste jusqu'en 1906.

De 1903 à 1906, Roussy avait publié non moins de 19 études traitant surtout de questions relatives à la pathologie et à la clinique du système nerveux ${ }^{8}$. En 1906, il devint lauréat des Hôpitaux de Paris (prix de l'Internat, médaille d'argent). La même année il fut nommé chef des Travaux de physiologie pathologique aux Hautes Etudes (Collège de France), fonction qu'il garda jusqu'en 1908.

Définitivement établi en France, Roussy opta en 1906 pour la nationalité française, recouvrant ainsi la citoyenneté originelle de sa famille, sans cesser, de ce fait, d'entretenir les relations qui le liaient à Vevey.

Le 20 février 1907, Roussy soutient sa thèse pour le doctorat en médecine. Une première partie de ce gros travail de 349 pages est consacrée à l'anatomie, à la physiologie et à la pathologie expérimentale de la «couche optique» («Sehhügel» des auteurs de langue allemande); la seconde partie se compose d'une étude histologique et pathologique du «syndrome thalamique» qui fut observé pour la première fois par Dejerine et Egger en 1903 et dont la dénomination fut proposée par Thomas et Chiray une année plus tard ${ }^{9}$. Roussy réunit dans sa thèse les quelques cas publiés antérieurement avec ses propres observations tant expérimentales que cliniques et donna de ce grave et affligeant syndrome une description devenue classique. En voici les symptômes selon le texte authentique de Roussy:

«Il s'agit de malades qui habituellement sans ictus font une hémiplégie motrice légère passagère et rétrocédant rapidement, sans trépidation épileptoïde, le plus souvent sans signe de Babinski. Cette hémiplégie s'accompagne de troubles de la sensibilité subjective et objective: subjective, ce sont des douleurs du côté paralysé, vives et tenaces, ne cédant à aucun traitement et constituant par elles-mêmes une réelle impotence (hémiplégie douloureuse); objective, c'est de l'hypoesthésie ou de l'anesthésie tactile, douloureuse et thermique, avec dysesthésie, paresthésie et topoesthésie; quelquefois de l'hyperesthésie, - enfin des troubles persistants de la sensibilité profonde, de la perte du sens musculaire, de l'astéréognosie et de l'hémiataxie. Souvent aussi apparaissent des mouvements choréo-athétosiformes. L'hémianopsie peut se rencontrer dans les cas de lésion intéressant la partie postérieure et inférieure de la couche optique. Tel est le tableau clinique du syndrome thalamique pur, d'après les cas cliniques que nous rapportons dans ce mémoire. ${ }^{10}$

La thèse de Roussy, qui est encore citée aujourd'hui, fut favorablement reçue par le jury présidé par le professeur Dejerine qui, avec sa femme, Madame 
Dejerine-Klumpke ${ }^{11}$, maître de laboratoire à l'Hospice de la Salpêtrière, avait été le grand patron de Roussy ${ }^{12}$.

La carrière de Gustave Roussy fut prestigieuse. Nous le retrouvons en 1908 préparateur d'anatomie pathologique à la Faculté de médecine de Paris où, la même année, il fut promu chef des Travaux pratiques d'anatomie pathologique. Lauréat de l'Institut (Académie des Sciences, prix Lallemand) en 1909, il fut reçu professeur agrégé de la Faculté de médecine en 1910.

L'année 1913 décidera de la future vocation de médecin et de savant de Gustave Roussy. C'est cette année-là qu'il fut nommé médecin en chef de l'Hospice départemental Paul-Brousse qui venait d'être fondé à Villejuif. Roussy «développa tout de suite le rayonnement de cet asile réservé aux vieillards, aux infirmes, à tous ceux enfin que la vie a marqués d'une tare physique irréparable. Non seulement chaque malade ou vieillard, à son admission, était l'objet d'un examen direct, mais encore ceux-ci devaient être groupés en familles pathologiques auxquelles les soins pouvaient être mieux et plus facilement assurés. ${ }^{13}$ Roussy avait done, il y a plus d'un demi-siècle, des idées tout à fait modernes au sujet de l'environnement des hospitalisés à long terme. Mais c'est surtout à l'Hospice de Villejuif, en apportant ses soins à tous ces pauvres hères qui remplissaient les salles de cette institution, laquelle était souvent le dernier refuge des personnes âgées ou incurables, que Roussy appris à connaître le vrai visage du cancer, ce «fléau social», ainsi qu'il s'exprime dans un des ses travaux ${ }^{14}$.

Survient la première guerre mondiale. Roussy est mobilisé. Il sert comme médecin aide-major de $1^{\text {re }}$ classe dans l'Ambulance 16/12. Gravissant les échelons de la hiérarchie des Services de santé, il devient médecin chef du Centre neurologique de la $\mathrm{X}^{\mathrm{e}}$ Armée, puis du Détachement de l'Armée de Lorraine. Vers la fin de la guerre on le voit médecin chef du Centre neurologique de la VII ${ }^{\mathrm{e}}$ Région à Besançon. Par ses emplois dans les services hospitaliers militaires, Roussy avait largement l'occasion d'observer de près les blessés du système nerveux qui ne cessait de l'occuper. Une série de publications sur les traumatismes tant somatiques que psychiques occasionnés par les lésions du cerveau, de la moelle épinaire et des nerfs périphériques témoignent de ces expériences qui, si tragiques qu'elles soient, ont néanmoins contribué au développement de la neurologie et de la psychiatrie.

La guerre terminée, Gustave Roussy reprit sa fonction de directeur de l'Hospice Paul-Brousse. Cette charge lui permit d'observer de près les ravages provoqués par le cancer. Les cancéreux de l'Hospice ne constituaient, pourtant, qu'une infime partie de tous ces malades atteints d'une des plus graves affections qui soient. Roussy se rendait bien compte qu'il existait trop de «cancéreux chroniques 
inopérables», des «malheureux qui meurent encore chez eux, dans des taudis, sans le secours que leur doit la société moderne $» .^{15}$ Animé par un esprit profondément humanitaire, il entreprit une vaste campagne en faveur de ces pauvres infirmes abandonnés et dépourvus de tous soins. La France, a cette époque - c'est Roussy qui le signale dans une importante conférence donnée au début de l'année 1921 à l'Union des Femmes de France - était «restée très en retard » dans la lutte anticancéreuse: il n’y avait «rien d'officiel [...] comme service hospitalier, jusqu'à la guerre où il n'existait, à Paris, que le petit hôpital de l'œuvre du Calvaire, dirigé par le docteur Récamier »; tout récemment, l'hôpital Pasteur venait de consacrer «quelques lits au traitement du cancer»; l'assistance publique avait bien ouvert, «à Brévannes, un service de cancéreux», mais ce service était «dépourvu de toutes ressources, de tout moyen d'action». ${ }^{16}$

Telle était selon les indications de Roussy la situation d'après-guerre en France pour les services anticancéreux spécialisés. Mais Gustave Roussy était précisément l'homme d'action qu'il fallait: aussi, dès que se furent apaisées les secousses sociales que la première guerre mondiale avaient déclenchées, le voyons-nous donner dans une modeste salle de l'Hospice Paul-Brousse une consultation spéciale réservée aux malades suspects d'être cancéreux ou qui redoutaient d'être porteurs de tumeurs. Il examinait lui-même chaque patient de tout âge, de toute condition, anxieux d'être atteint de la maladie si redoutée; et c'était encore lui qui analysait chaque préparation biopsique. Dans ces premières consultations, le dépistage précoce du cancer constituait déjà une des fins principales.

Grâce à son rayonnement, le centre de Villejuif recrutait un nombre de plus en plus considérable de malades et se transformait également en un organisme d'enseignement tout ensemble scientifique et clinique. Roussy avait ainsi réussi à créer, avec l'autorisation du Conseil général de la Seine, dès 1921 au sein d'un asile de vieillesse dont la destination était tout autre, un organisme nouveau qui prit, par la suite, le nom de «Centre Anticancéreux de la Banlieue Parisienne». Les moyens d'action de ce service étaient pourtant assez restreints: «Quelques lits, une installation radiothérapeutique, le service de chirurgie de l'hospice, un laboratoire aux ressources des plus limitées, telle fut l'organisation primitive qui devait bientôt donner naissance à l'Institut du Cancer de Villejuif. » ${ }^{17}$

En effet, les projets de Roussy visaient plus haut. C'est ici qu'il convient de se poser la question de savoir si, dans l'élaboration de ses vastes projets, Roussy ne s'est pas inspiré de ses excellentes connaissances qu'il possédait de l'histoire de la médecine, qui ne cessa de l'intéresser. On le voit se référer expressément à d'anciennes tentatives dans la lutte anticancéreuse qui, pourtant, n'avaient guère trouvé l'écho que l'importance du problème requérait. 
«C'est en Angleterre qu'à la fin du XVIII ${ }^{\mathrm{e}}$ siècle, s'est fondé le premier organisme central ayant pour but de lutter contre le cancer et de créer un mouvement pouvant être l'origine d'une campagne anticancéreuse. Il est intéressant de relever dans les documents du Middlesex-Hospital (à Londres), déjà anciens, puisqu'ils datent du 19 janvier 1792, les principaux éléments de la lutte anticancéreuse. Ils ne diffèrent guère de ceux de nos organisations modernes.

Le médecin anglais Howard, voulant créer un centre de recherches et d'études pour le cancer, avait posé les principaux éléments de cette campagne. Il se proposait:

D'accorder un asile définitif aux malades incurables;

D'établir une consultation pour traiter les malades;

De créer un hôpital destiné à recevoir les malades;

D'essayer, dans des centres de recherches, d'appliquer les méthodes de traitement;

Et enfin de créer des centres de recherches où l'on pourrait poursuivre l'étude de la cause du cancer.

Nous trouvons, dans cette première charte de l'organisation de la lutte anticancéreuse, tous les éléments qui plus tard ont été repris et développés dans les différents pays d'Europe et d'Amérique. ${ }^{18}$

S'inspirant de ces principes, Roussy se voua à leur réalisation. Les modestes installations du Centre anticancéreux qu'il avait mis sur pied dans l'Hospice Paul-Brousse à Villejuif ne répondaient guère aux idées qu'il se faisait du combat à mener contre le cancer. Ses efforts pour intensifier cette lutte furent enfin couronnés de succès: en décembre 1925, à son instigation, les pouvoirs publics votèrent les sommes nécessaires destinées à jeter les bases de l'Institut pour l'Etude et le Traitement du Cancer. A la même époque fut créée une Fondation pour le développement de l'Institut du Cancer de la Faculté de médecine de Paris; elle fut reconnue d'utilité publique par décret du 26 novembre 1927. Son but principal était d'assurer le fonctionnement scientifique et matériel de l'Institut du Cancer dont les laboratoires ont été terminés le $1^{\text {er }}$ décembre 1929 et inaugurés le 27 mars 1930 par Gaston Doumergue, président de la République française. A cette occasion fut posée la première pierre de la section hospitalière qui devait comprendre 150 lits. Elle fut ouverte le $1^{\text {er }}$ mars 1934 sous la direction de Roussy; l'inauguration eut lieu le 16 juin 1934 par le président de la République, Albert Lebrun ${ }^{19}$.

L'Institut du Cancer poursuivait un triple but: être tout à la fois un organisme scientifique, un organisme hospitalier et un organisme d'enseignement et de propagande $^{20}$.

Gustave Roussy avait été promu professeur d'anatomie pathologique en 1925. Il fut élu doyen de la Faculté de médecine de Paris en 1933, ce qui l'incita à s'occuper davantage de questions déontologiques et de la formation profession- 
nelle des futurs médecins. En 1937, il fut nommé par le Gouvernement recteur de l'Université de Paris, devenant ainsi le premier médecin à occuper cette fonction d'une importance capitale pour la vie intellectuelle de toute la France ${ }^{21}$.

Malgré les charges que lui imposaient ses fonctions de directeur de laboratoire et de chef de clinique, Roussy ne cessa pas d'être un des médecins les plus remarquables par ses travaux scientifiques. En 1939, un catalogue critique de ses publications comporte près de 300 titres $^{22}$; encore cette liste est-elle incomplète. Ses recherches s'étendent sur toute la pathologie, en mettant l'accent sur le système nerveux et neuro-endocrinien; mais ce sont surtout les tumeurs, bénignes ou malignes, qui retinrent son attention. Son Précis d'anatomie pathologique écrit en collaboration avec Roger Leroux et Charles Oberling (2 vol., Paris, 1933) est non seulement un témoignage de l'intérêt qu'il portait à la pathologie générale et spéciale, mais prouve aussi à quel point l'enseignement de la discipline qu'il représentait lui tenait à cœur dans l'instruction médicale.

Prodige d'activité, Roussy, membre et secrétaire général de l'Académie des sciences, fut un des principaux promoteurs et animateurs de l'Association française pour l'étude du cancer. Dès la création de cette société (1908), il en fut un des membres les plus actifs. Elu secrétaire général en 1922, il le resta pendant quatorze ans; en 1937 il en devint le président ${ }^{23}$.

Chez Roussy, la science primait la croyance: "Sur le plan philosophique, Gustave Roussy admettait sans contrainte, que d'autres possédassent une foi qu'il ne partageait pas. 'Nous ne nions pas, bien entendu, écrivait-il, la légitimité des croyances religieuses, nous cherchons seulement à interpréter les données de l'expérience en faisant la plus grande économie possible de notions a priori'. » ${ }^{24}$ Aussi, «sa discipline cartésienne le rendait habile à discerner le vrai dans le progrès des innovations thérapeutiques. ${ }^{25}$

La solidité scientifique et l'originalité de ses travaux valurent à Roussy beaucoup de titres honorifiques. La Suisse, son pays d'origine, semble pourtant l'avoir oublié, Genève mise à part. Il fut nommé membre correspondant de la Société médicale de Genève et y fut reçu docteur honoris causa en 1936.

Comme on le sait, Gustave Roussy ne s'était pas uniquement livré à la recherche scientifique; il fut aussi médecin et chef de clinique. Selon le souvenir du professeur René Huguenin, son ancien collaborateur et son successeur à la direction des Services hospitaliers de l'Institut du Cancer, l'attitude de Roussy à l'égard de ses patients était emprunte d'un dévouement exemplaire.

«Dans sa vie hospitalière, son cœur était toutes nuances de douceur, comme son esprit tout de nécessaire fermeté. Dès la première consultation, le malade, assis face à lui, ne voyait plus rien d'autre qu'un regard affectueusement curieux de son mystère, un 
sourire penché vers ses angoisses, une main tendue pour son secours. Le désespéré avait cessé de se sentir un grand malade, pour n'être plus qu'un homme apeuré; et, quelle que pût être la fin de son drame, toujours il partait rassuré sur un favorable avenir. Nanti d'une prescription d'espérance, le patient était bientôt disposé à accepter, sinon à réclamer, les thérapeutiques les plus longues et les plus pénibles, qui l'eussent rebuté dès l'abord. [...] Jamais assez satisfait des récits que lui faisaient ses assistants sur l'efficience des prescriptions édictées, il s'en allait de temps à autre, en tapinois, pour assurer le patient qu'il ne l'oubliait pas, que les jeux étaient bien menés, et surtout qu'il n'y avait pas assez de péril pour que s'imposât quelque bouleversement thérapeutique. Simplement, comme l'ami qui vient aux nouvelles, le Chef s'asseyait au pied du lit, pour apaiser les ressauts d'inquiétude et ranimer l'espoir menacé. ${ }^{26}$

De nos jours encore, on peut rencontrer des personnes qui, en termes émus, évoquent l'inlassable générosité et la bonté de cœur que Roussy, souvent le dernier espoir, avait jadis prodiguées à leurs parents atteints d'un cancer.

Gustave Roussy avait épousé $\mathrm{M}^{\text {lle }}$ Thomson dont le père fut ministre. Le ménage n'eut pas d'enfant.

En raison de son âge, Roussy démissionna de sa fonction de directeur de l'Institut du Cancer le 22 janvier $1946^{27}$.

Les dernières années de Gustave Roussy furent tragiques.

Dans un éloge rédigé en 1949, Jean Lhermite, le collègue de Roussy à l'hospice Paul-Brousse, avait écrit: «Mais, déjà des forces mauvaises se dressaient contre l'autorité du Recteur; il m'en fit confidence tout en les méprisant, et c'est à une conjuration dont il me faut taire les mobiles que Gustave Roussy, sous le prétexte d'une manifestation d'étudiants, fut révoqué de ses fonctions. C'était le 11 Novembre $1940 .{ }^{28}$

En effet, le 11 novembre 1940, anniversaire de l'Armistice de la première Guerre, les étudiants parisiens, portant des cannes à pêche en guise de gaules (!), manifestèrent place de l'Etoile, auprès de la tombe du Soldat Inconnu. Roussy, recteur de l'Université, ne s'étant pas opposé à ces manifestations, fut sur-lechamp destitué de ses fonctions. Cependant, «dès la libération du Territoire, il était réintégré dans le poste éminent dont il avait été injustement destitué ». ${ }^{29}$

Mais le 29 mai 1947 on put lire, et en grosses lettres, dans les journaux que le Conseil des ministres, sous la présidence de Vincent Auriol, président de la République, venait de suspendre Gustave Roussy de ses fonctions et qu'une instruction judiciaire avait été ouverte sur la plainte du ministre des Finances, Robert Schumann. Ce scandale qui occupa vivement la presse pendant près de deux semaines, compromettait gravement non seulement Gustave Roussy, «mais aussi l'Université de Paris dont il était le chef vénéré, et l'Université française tout 
entière. [...] Notre dignité, notre honneur étaient en jeu. Atteindre le chef, c'était atteindre le corps professoral tout entier». ${ }^{30}$ "L'affaire Roussy», pour reprendre la formule de la langue journalistique, n'a pourtant, à notre connaissance, jamais été éclaircie. Nous nous en tenons à ce qu'en écrivait en 1977 le Professeur Pierre Denoix: «Un scandale politico-financier aux dessous douteux s'efforça de compromettre l'homme que nous venons d'évoquer [Roussy] et si rien n'aurait dû mettre en doute son intégrité, la malignité de quelques hommes finit par atteindre leur but.» ${ }^{31}$

Renonçant finalement «à la lutte injuste et cruelle que lui avaient imposée ses adversaires avoués ou masqués, et, en un suprême et tragique geste de protestation $"{ }^{32}$ Gustave Roussy se donna la mort jeudi le 30 septembre $1948^{33}$. Mais moins de deux ans plus tard, il fut officiellement réhabilité: par décret du $1^{\text {er }}$ avril 1950 l'Institut du Cancer reçut le nom de son fondateur: Institut Gustave-Roussy (Centre Clinique et Thérapeutique) ${ }^{34}$. Et le vendredi 2 mars 1951 s'est déroulée à cet Institut Gustave-Roussy une cérémonie officielle dont le ministre de la Santé publique et de la Population avait accepté la présidence. En présence de $\mathbf{M}^{\text {me }}$ Gustave Roussy et d'une nombreuse assistance, des allocutions furent prononcées par les autorités civiles et universitaires. Deux médaillons portant le profil de Roussy avaient été apposés sur les deux sections de l'Institut. A l'issue de la cérémonie, ils furent dévoilés par le professeur Laubry, membre de l'Académie de médecine et de l'Académie des sciences, qui représentait les amis et les élèves de Roussy ${ }^{35}$.

Gustave Roussy s'était distingué dans beaucoup de domaines. C'est pourtant par sa lutte contre le cancer qu'il s'est acquis les plus grands mérites. Ce fut son ancien collaborateur Charles Oberling, professeur à la Faculté de médecine et directeur de l'Institut de recherches scientifiques sur le cancer qui, dans son allocution prononcée à la cérémonie du 2 mars 1950, retraça les qualités de Roussy à ce sujet: «Rien de tout cela n'existait il y a trente ans. A ce moment, l'étude du cancer était considérée comme une cause perdue. Lui consacrer son activité, c'était gâcher son temps. Chercher des résultats, c'était poursuivre des chimères. Malgré cela, Gustave Roussy s'est lancé dans cette lutte corps et âme. Etait-ce l'attrait de la difficulté, l'immensité du problème conduisant dans tous les domaines de la biologie? Tout cela a certainement compté, mais ceux qui l'ont connu savent qu'en dehors des considérations d'ordre intellectuel, des facteurs humains ont toujours eu beaucoup de prise sur ses déterminations [...] Voilà les mobiles qui l'ont engagé dans cette lutte contre le cancer qui, rapidement, allait devenir le centre de toute son activité, l'objet principal de ses préoccupations. ${ }^{36}$ 
Nous ne saurions mieux terminer la présente esquisse biographique sur celui qui fut dans sa jeunesse notre compatriote qu'en résumant par ses propres paroles les principes qui le guidaient dans ses recherches sur le cancer. Selon Charles Oberling, Gustave Roussy avait inlassablement insisté sur le fait que «l'étude du cancer n'est plus, comme elle le fut pendant longtemps, l'apanage des sciences morphologiques, mais qu'elle étend son domaine d'investigations à toutes les branches de la biologie, qu'elle utilise tour à tour les méthodes et les données nouvelles que nous apportent les progrès de la chimie, de la chimie-physique, de la pathologie expérimentale et comparée ». ${ }^{37}$ Ces idées de Roussy sont encore aujourd'hui valables.

A l'heure actuelle un nouvel Institut Gustave-Roussy, conçu sur des plans tout modernes dans lesquels entrent pourtant les idées principales de Roussy, est en cours d'achèvement au lieu-dit Hautes-Bruyères à Villejuif ${ }^{38}$.

\section{Notes}

${ }^{1}$ Il n'existe pas de biographie intégrale de Gustave Roussy. Nous sommes, par conséquent, d'autant plus reconnaissant au Professeur Pierre Denoix des nombreux renseignements qu'il a bien voulu nous donner.

Voici la liste des ouvrages (avec les sigles employés dans la présente étude) que nous avons eu l'occasion de consulter.

a) Denoix, Pierre:

- Originalité des Centres anticancéreux français, Institut Gustave-Roussy, 1962. Sigle: Centres anticancéreux.

- Gustave Roussy, 1977. (Cette étude sur Roussy a paru en septembre 1977 dans le bulletin «Informations» de l'Institut Gustave-Roussy qui n'est diffusé que parmi le personnel de la maison.)

Sigle: Denoix.

b) Mocquot, Pierre:

- Hommage à la mémoire de Gustave Roussy. Bulletin du Cancer, t. 37, 1950, pp. $80-82$.

Sigle: Mocquot.

c) Roussy, Gustave:

- La Couche optique (Etude anatomique, physiologique \& clinique) - Le Syndrome thalamique. Thèse pour le doctorat en médecine, présentée et soutenue le mercredi 20 février 1907, à 1 heure. Sigle: Thèse.

- Le Cancer fléau social. Conférence donnée à l'Union des Femmes de France le 24 février 1921, Paris, 1921.

Sigle: Le Cancer.

- Titres et travaux scientifiques du $D^{r}$ Gustave Roussy, Paris, 1924.

Sigle: Travaux. 
- Titres et travaux scientifiques du $D^{r}$ Gustave Roussy, Supplément, 1929, Paris, 1929. Sigle: Supplément.

- Notice sur les titres et travaux scientifiques de M. Gustave Roussy, Paris, 1939. Sigle: Notice.

d) Autres publications:

- Hommage à Gustave Roussy, Paris, 1951. (Il s'agit de discours prononcés par les autorités à l'Institut du Cancer le 2 mars 1951 à l'occasion du dévoilement de 2 médaillons représentant Gustave Roussy.)

Sigle: Hommage.

- Description et fonctionnement de l'Institut Gustave-Roussy, Université de Paris, s.d. Sigle: Description et fonctionnement.

- Institut Gustave-Roussy Hautes-Bruyères, J.G.R., Villejuif, 1977.

Sigle: I.G.R.

${ }^{2}$ Denoix, p.1.

${ }^{3}$ Dans la dédicace de sa Thèse, Roussy ne manque pas de citer ses «premiers Maîtres de la Faculté de médecine de Genève, MM. les professeurs Éternod, Laskowski et Prévost».

${ }^{4}$ Travaux, p.9. - Il convient de donner ici quelques indications concernant la biographie de ces professeurs qui avaient enseigné Roussy: a) François-Franck (Charles-EmileAlbert), né à Paris en 1849. Sa vie scientifique s'écoula au Collège de France où il fut professeur de physiologie. Il s'appliqua à l'étude de la physiologie normale et pathologique de la circulation sanguine et du système nerveux autonome. Membre de l'Académie de médecine depuis 1887, il mourut à Paris en 1921. - b) Darier (Jean), né à Genève en 1856 et mort à Longpont (aux environs de Paris) en 1938. Médecin à l'hôpital Broca, où fonctionnait un service de dermato-vénéréologie, il devint par la suite un éminent dermatologue. Il fut aussi le secrétaire général de l'Association française pour l'étude du cancer. En 1919 il fut reçu membre de l'Académie de médecine. On le connaît surtout par la maladie qu'il a décrite («maladie de Darier») qui est une dyskératose bénigne. c) Dejerine (Jules) avait, comme Darier et Roussy, vu le jour en Suisse: il naquit à Genève en 1849. De bonne heure à Paris, il y devint docteur en 1879, agrégé en 1886, professeur en 1901 et membre de l'Académie de médecine en 1908. Il succéda à la Salpêtrière à Raymond qui avait remplacé Jean-Martin Charcot. Ses nombreux travaux ont porté en premier lieu sur le système nerveux. Il mourut à Paris en 1917. - d) Marie (Pierre), né à Paris en 1853. Elève de Charcot, il devint professeur agrégé en 1889 et titulaire de la chaire de clinique neurologique de la Salpêtrière en 1918. Il s'est distingué par ses nombreux travaux sur la pathologie du système nerveux et musculaire. Chef inventif, il a contribué à la formation de beaucoup de neurologues. Il fut reçu membre de l'Académie de médecine en 1911. Il mourut à Cannes en 1940.

5 Ibid., p. 9.

${ }^{6}$ Ibid., p. 9.

7 Nous empruntons les dates de la biographie de Roussy à la liste des titres qu'il a obtenus et des fonctions qu'il a assumées; elle se trouve en tête de sa Notice, p.V-VII.

${ }^{8}$ On trouvera la liste des travaux médicaux (avec les références) de Roussy à la fin de sa Notice, p.123-142. 
${ }^{9}$ Thèse, p.192-193. Il convient de remarquer que les parties principales de la Thèse sont introduites par un aperçu historique ce qui témoigne de la précocité de l'intérêt que Roussy portait à l'histoire de la médecine.

10 Thèse, p. 210-211.

${ }^{11} \mathbf{M}^{\mathrm{me}}$ Dejerine, née Augusta Klumpke (1859-1927), avait vu le jour à San Francisco. Après ses études à Lausanne et à Paris, elle fut la première femme à occuper la fonction d'externe (1882) et d'interne (1886) dans un hôpital. Neurologue distinguée, elle décrivit en 1885 la paralysie du plexus brachial qui porte son nom, "paralysie de Klumpke», «Klumpkesche Lähmung». Elle a été pour son mari une précieuse collaboratrice. Ce fut Roussy qui fit son éloge, Revue Neurologique, $\mathrm{n}^{\circ}$ 6, juin 1927.

12 C'était Jules Dejerine qui, avec Pierre Marie, avait porté le jeune Gustave Roussy à se livrer à l'étude de la couche optique et du syndrome thalamique (Thèse, p.4).

13 Denoix, p. 1.

${ }^{14}$ Le Cancer, page de titre.

${ }^{15}$ Le Cancer, p. 13.

${ }^{16}$ Le Cancer, p. 13.

17 Denoix, p. 2.

${ }^{18}$ Le Cancer, p.12-13. - Le professeur Erwin H.Ackerknecht, dans son étude, Geschichte und Geographie der wichtigsten Krankheiten, Stuttgart, 1963, donne à la page 153 quelques autres détails: "Bereits 1782 wurde ein 'Cancer Ward' im Middlesex-Hospital eröffnet, und 1802 wurde in London eine 'Gesellschaft zur Erforschung der Natur und der Kur des Krebses' von prominenten Chirurgen wie M.Baillie, R. Willan, J.Abernethy und andern gegründet, die allerdings nach einigen Jahren wieder einging.»

${ }^{19}$ Pour des questions de détail on consultera le travail de Gustave Roussy, «La lutte sociale contre le cancer dans le département de la Seine (Banlieue parisienne) et dans quelques départements voisins», Bulletin de l'Académie de Médecine, $\mathrm{n}^{\circ}$ 14, 16 avril 1929, également publié dans la Notice, p.38-42. - Cf. aussi Centres anticancéreux.

20 Notice, p. 40.

${ }^{21}$ Denoix, p. 4.

${ }^{22}$ Remarquons que Roussy donne dans ses ouvrages, Titres et travaux, Supplément et Notice, en plus d'un catalogue de ses publications un vaste résumé de ses travaux.

${ }^{23}$ Cf. à ce sujet Mocquot.

${ }^{24}$ Denoix, p. 5.

25 «Allocution prononcé par Monsieur le Professeur René Huguenin», Hommage, p.17.

${ }^{26}$ Ibid., p.16-17.

${ }^{27}$ La succession de Roussy fut confié au professeur René Huguenin qui devint le premier titulaire de la Chaire de cancérologie médicale et sociale de la Faculté en 1951. Après la disparition prématurée de René Huguenin, le docteur Pierre Denoix fut nommé directeur le 10 février 1956. Il nous faut encore préciser que suivant une ordonnance de 1945, la section scientifique de l'Institut du Cancer fut transférée sous l'autorité du Centre National de la Recherche scientifique et placée sous la direction du professeur Charles Oberling. Au sujet de ces questions historiques, on consultera «Les étapes de la création de l'Institut », Description et fonctionnement, p.3-5, et Centres anticancéreux.

${ }^{28}$ La citation est empruntée à Denoix, p.5.

${ }^{29}$ Ibid., p. 5. 
30 «Allocution prononcée par Monsieur le Recteur Jean Sarrailh, Président du Conseil de l'Université de Paris», Hommage, p.8. (Le «Président du Conseil» est le recteur de l'Université.)

${ }^{31}$ Denoix, p. 5 .

${ }^{32}$ Jean Sarrailh, loc. cit., p. 7.

${ }^{33}$ Le Figaro, no 1263 du Samedi/Dimanche, 2-3 octobre 1948, $122^{\mathrm{e}}$ année, première page.

${ }^{34}$ Description et fonctionnement, p. 4 .

${ }^{35}$ La plaquette Hommage donne de cette manifestation un rapport circonstancié.

${ }^{36}$ "Allocution prononcée par Monsieur le Professeur Charles Oberling», Hommage, p. 21.

${ }^{37}$ La citation de Roussy est rapportée par Charles Oberling, ibid., p.21.

${ }^{38}$ C.. I.G.R. et Centres anticancéreux.

\section{Summary}

Gustave Roussy was born in 1874 in Vevey, Switzerland. He studied in Geneva and Paris, where he made a rapid and brilliant medical career. As chief Medical Officer of the Paul Brousse Hospice in Villejuif near Paris in 1913 he emphasized the clinical and social aspects of cancer. It was here that he founded in 1921 the Institute of Cancer which he never ceased to support with generous donations which helped the soaring success of the Institute.

Gustave Roussy was awarded his Doctor of Medicine degree in 1907 for his work on the thalamic syndrome. In 1910 he was named Professor on the Faculty of Medicine in Paris, Dean in 1933 and Rector of the University in 1937. Roussy died in 1948 among obscure but tragic circumstances which were never clarified. However he was rehabilitated officially in 1950. It was on this date too that the Institute of Cancer - by ministerial decree - received its new name of Institute Gustave Roussy.

$\mathrm{D}^{\mathrm{r}}$ med. et phil. René Bernoulli

Ehrendozent à la Faculté de médecine

35, Amselstrasse

CH-4059 Bâle 